\title{
FINITE SIMPLE GROUPS WITH NILPOTENT THIRD MAXIMAL SUBGROUPS
}

T. M. GAGEN and Z. JANKO

(Received 29 September 1965)

We say that a subgroup $H$ is an $n$-th maximal subgroup of $G$ if there exists a chain of subgroups $G=G_{0}>G_{1}>\cdots>G_{n}=H$ such that each $G_{i}$ is a maximal subgroup of $G_{i-1}, i=1,2, \cdots, n$. The purpose of this note is to classify all finite simple groups with the property that every third maximal subgroup is nilpotent.

THEOREM. If $G$ is a finite simple group such that every third maximal subgroup of $G$ is nilpotent, then $G$ is isomorphic either to a linear fractional group PSL $(2, q)$, for certain $q>3$, or to $S z\left(2^{3}\right)$, the Suzuki simple group over the field of $2^{3}$ elements.

REMark. If the group $P S L(2, q)$ satisfjes the condition that all third maximal subgroups are nilpotent then it follows that

(a) $q=2^{r}, 3^{s}$ or $t$, where $r, s, t$ are primes, $r>2$, and

(b) if $q=3^{r}$ or $2^{s}$, then $(q+1) / \varepsilon,(q-1) / \varepsilon$, where $\varepsilon=2$ if $q$ is odd, and $\varepsilon=1$ if $q$ is even, are products of at most two (not necessarily distinct) primes; if $q=t$, then $(t-1) / 2$ is a product of at most two primes and $(t+1) / 2$ is either a product of at most two primes or a power of 2.

Conversely, the groups $S z\left(2^{3}\right)$ and $P S L(2, q)$, where $q$ satisfies the conditions (a) and (b) above, have the property that every third maximal subgroup is nilpotent.

Notations AND KNOWN RESUlts. We let $H \leqq G, H<G, H \unlhd G$ mean that $H$ is a subroup, a proper subgroup, and a normal subgroup of $G$, respectively. We let $N(S), C(S)$, for any subset $S$ of $G$, denote the normalizer and the centralizer of $S$ in $G$, respectively. We let $Z(G)$ denote the centre of $G$. If $x$ is any element of $G$ we let $\langle x\rangle$ be the group generated by $x$.

The following result of Janko [3] and Berkovič [1] is essential.

LEMMA 1 . If $G$ is a finite group all of whose second maximal subgroups are nilpotent, then $G$ is either soluble or isomorphic to $P S L(2,5)$ or $S L(2,5)$. 
Proof of Theorem. According to a definition of J. G. Thompson [6], we say that a finite group $G$ is an $N$-group if the normalizer of any nontrivial soluble subgroup of $G$ is itself soluble.

If $G$ is a non-abelian simple group all of whose third maximal subgroups are nilpotent, then $G$ is an $N$-group. For suppose that there exists a non-trivial soluble subgroup $S \leqq G$ such that $N(S)$ is non-soluble. The group $H=N(S)$ is clearly a maximal subgroup of $G$. For if not, there exists a subgroup $M$, with $H \leqq M$, which is second maximal in $G$. But then $M$ has the property that every proper subgroup of $M$ is nilpotent, and hence, by a result of Iwasawa [2], we see that $M$ is soluble, a contradiction. Now $H$, being maximal in $G$, has the property that every second maximal subgroup of $H$ is nilpotent. By Lemma 1 we see that $H \cong P S L(2,5)$ or $H \cong S L(2,5)$. Since $S \neq 1$, we have that $H \cong S L(2,5)$ and $S=Z(H)$. The Theorem D of Suzuki [5] p. 682, gives that $G$ is non-simple, a contradiction.

Therefore $G$ is a $N$-group. Now Thompson [6] has classified all finite $N$-groups and since $G$ is simple we see that $G$ is isomorphic to one of the following groups:

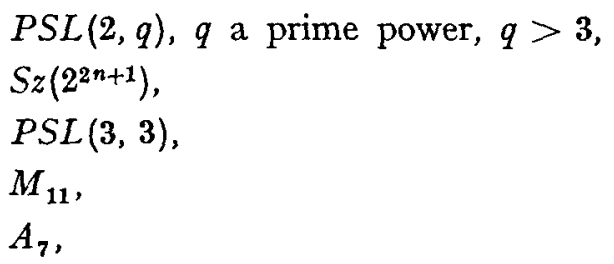

Case 1. The groups $S z(q), q=2^{2 n+1}, n \geqq 1$.

Let $2 q=r^{2}, r=2^{n+1}$. Then if $G=S z(q)$, the following relations must be satisfied:

$$
\begin{aligned}
q-1 & =\text { prime } \\
q+r+1 & =\text { prime } \\
q-r+1 & =\text { prime. }
\end{aligned}
$$

These are satisfied only if $n=1$, since at least one is divisible by 5 .

Case 2. The group $\operatorname{PSL}(3,3)$ is inadmissible since this contains the Hessian group $H$ as a subgroup. The group $H$ has subgroups $H>F>E$, in the notation of Miller-Blichfeldt-Dickson [4] p. 239 and $E$ is non-nilpotent of order 36 .

Case 3. The groups $M_{11}$ and $A_{7}$.

Both these groups are inadmissible since they contain a subgroup 
isomorphic to $A_{6}$, while non-soluble subgroups of any group with our property are either $P S L(2,5)$ or $S L(2,5)$.

Case 4. The group $\mathrm{PSU}_{3}\left(3^{2}\right)$.

This group is inadmissible since $P S U_{3}\left(3^{2}\right)>U_{2}\left(3^{2}\right)$. The group $S U_{2}\left(3^{2}\right)$ is contained in $U_{2}\left(3^{2}\right)$ with index 4 and is non-nilpotent.

Thus we have ruled out all possibilities except the linear fractional groups and the group $S z\left(2^{3}\right)$, as stated in the theorem.

Now suppose that the group $G$ is isomorphic to $\operatorname{PSL}(2, q), q>3$. Then $G$ has the property that every third maximal subgroup is nilpotent if and only if every maximal subgroup $H$ has the property $(*)$ every second maximal subgroup of $H$ is nilpotent.

Let $q=p^{n}>3, p$ a prime. A $p$-Sylow normalizer $N$ is isomorphic to the groups of transformations of $G F(q)$

$$
x \rightarrow \alpha^{2} x+\beta, \quad \alpha, \beta \in G F(q), \quad \alpha=0 .
$$

It follows that $N$ satisfies (*) if and only if

(a) $(q-1) / \varepsilon$ is a product of less than or equal to two primes (not necessarily distinct);

(b) $n=1$ when $p>3$;

(c) $n$ is a prime greater than 2 when $p=3$;

(d) $n$ is a prime when $p=2$.

The dihedral subgroup $D_{2(a+1) / \varepsilon}$ satisfies $(*)$ if and only if

(e) $(q+1) / \varepsilon$ is a product of at most two primes or a power of 2 .

The condition (a) implies that $(*)$ holds for the dihedral groups $D_{2(a-1) / \varepsilon}$. The only other possible maximal subgroups are $P S L(2,5)$, $\operatorname{PSL}(2,3), S_{4}$ or $\operatorname{PSL}(2,2)$, and these groups automatically satisfy $(*)$.

\section{References}

[1] Ja. G. Berkovic, 'The existence of subgroups of a finite non-soluble group', Dokl. Akad. Nauk 156 (1964), 1255-1257.

[2] K. Iwasawa, 'Uber die Struktur der endlichen Gruppen, deren echte Untergruppen sämtlich nilpotent sind', Proc. Phys. Math. Soc. Japan 23 (1941), 1-4.

[3] Z. Janko, 'Endliche Gruppen mit lauter nilpotenten zweitmaximalen Untergruppen', Math. Zeitschr. 79 (1962), 422-424.

[4] G. A. Miller, H. F. Blichfeldt and L. E. Dickson, Theory and applications of finite groups (New York 1938). 
[5] M. Suzuki, 'On finite groups with cyclic Sylow subgroups for all odd primes', Amer. $J$. Math. 77 (1955), 657-691.

[0] J. G. Thompson, 'Some simple groups', Symposium on group theory (Harvard, 1963).

Australian National University, Canberra

and

Monash University, Melbourne 